\title{
Dynamic Bayesian Networks for Real-Time Classification of Seismic Signals
}

\author{
Carsten Riggelsen, Matthias Ohrnberger, and Frank Scherbaum \\ University of Potsdam, Institute of Geosciences \\ Karl-Liebknecht-Str. 24/25, 14476 Golm, Potsdam, Germany \\ \{riggelsen, mao,fs\}@geo.uni-potsdam.de
}

\begin{abstract}
We present a novel method for automatic classification of seismological data streams, focusing on the detection of earthquake signals. We consider the approach as being a first step towards a generic method that provides for classifying a broad range of seismic patterns by modeling the interrelationships between essential features of seismograms in a graphical model. Through a continuous Wavelet transform the features are extracted, yielding a time-frequencyamplitude decomposition. The extracted features obey certain Markov properties, which allows us to form a joint distribution in terms of a Dynamic Bayesian Network. We performed experiments using real seismic data recorded at different stations in the European Broadband Network, for which we achieve an average classification accuracy of $95 \%$.
\end{abstract}

\section{Introduction}

Last decade's developments in data acquisition, data storage and data transmission technologies via internet allow seismologists to collect new data at an unprecedented rate. Still, the impact of recent large earthquakes (e.g. Kobe 1995, Sumatra 2004) demonstrates that the need for establishing even denser seismological network infrastructures will continue [10] in order to allow for detailed research on earthquake source processes and to enable the implementation of early warning systems for critical infrastructure facilities. Consequently, robust automatic algorithms have to be used for scanning data streams automatically in order to aid seismologists in extracting the relevant portions for further analysis.

The main purpose of existing seismic signal detection algorithms is the automatic detection and timing of body phase arrivals in seismogram recordings of tectonic earthquakes and artificial explosions (chemical or nuclear) — for a review, see [12]. Pattern recognition approaches, which aim to jointly detect and classify the complete seismogram, have rarely been used in the context of seismic signal detection and classification. In [6] a robust seismic event detector has been developed which is based on the comparison of spectral images (sonogram) to a set of reference templates. More recently, in [5] continuous seismogram recordings have been represented by using the discrete wavelet transform and de-noising techniques and then classified into local, regional and teleseismic events categories.

Within this study, we aim to demonstrate the use of Dynamic Bayesian Networks as a very general and symbolic approach to seismic signal classification. 


\section{Dynamic Bayesian Networks}

The graphical models framework is an attractive and intuitively appealing statistical formalism for modeling a broad range of real-life situations and systems. Bayesian networks (BN), a particular kind of graphical model, has been used extensively for modeling static systems. When modeling dynamic systems that produce data as time passes, it is beneficial to explicitly account for the temporal element in the model. This gives rise to so-called Dynamic Bayesian Networks (DBN), a generalistion of the less powerful Hidden Markov Models.

A BN [11] is a concise way of representing a joint probability distribution over variables $\boldsymbol{X}=\left\{X_{1}, \ldots, X_{n}\right\}$. Formally a BN consists of directed acyclic graph (DAG), where every vertex coincides with a variable $X_{i}$, and a set of conditional probability distributions (CPDs) $p\left(X_{i} \mid \boldsymbol{\Pi}\left(X_{i}\right)\right)$, where $\boldsymbol{\Pi}\left(X_{i}\right)$ is the set of parents of $X_{i}$ in the DAG. We use (conditional) multinomials and Gaussians as CPDs. The DAG encodes conditional independence restrictions for a joint distribution $\operatorname{Pr}(\boldsymbol{X})$ via the so-called Markov properties, such that we may write $\operatorname{Pr}(\boldsymbol{X})=\prod_{i} p\left(X_{i} \mid \boldsymbol{\Pi}\left(X_{i}\right)\right)$.

In a DBN [4] we are interested in $\boldsymbol{X}=\left\{X_{1}, \ldots, X_{n}\right\}$ at every point in time; to distinguish times, we write $X_{i}^{t}$ to refer to the $i$ th variable at time $t$. DBNs are time-invariant, such that the DAG of the overall DBN is a repeating structure, and the CPDs do not change with time. The joint distribution is then $\operatorname{Pr}\left(\boldsymbol{X}^{0}, \ldots, \boldsymbol{X}^{T}\right)=$ $\prod_{t=0}^{T} \prod_{i} p\left(X_{i}^{t} \mid \boldsymbol{\Pi}\left(X_{i}^{t}\right)\right)$ where the parents of the variables in slice $t$ may occur in slice $t$ (intra links) or $t-1$ (inter links) except for slice $t=0$, where only intra links exist. Figure 2 shows an example of a DBN. The repeating structure and the CPDs (shaded region) is unrolled until $T$; the structure and CPDs defined at time $t=0$ appears only once at the beginning. The variables $\boldsymbol{X}=\boldsymbol{U} \cup \boldsymbol{O}$ may be observed, $\boldsymbol{O}$, while other variables may be hidden, $\boldsymbol{U}$ (for unobserved).

\section{Model Specification}

In the next sections we develop a generic generative DBN for seismic waves. In Section 3.1 we start off by defining the feature space of wave patterns. The features are defined such that we explicitly can specify their (in)dependences via intra- and inter-frame links in the qualitative sense- - this we discuss in Section 3.2

\subsection{Continuous Wavelet Transform}

For the so-called feature extraction we depart from a Continuous Wavelet Transform (CWT) (see for instance [3]), a mathematical analysis technique perfectly suitable for describing cyclic sequences, multiscale features and in particular seismic signals [8].

In contrast to a normal Fourier transform, the Wavelet transform tells us not only what happens, but also when it happens, i.e., we obtain information localized in time and frequency. The CWT coefficients are the inner product of signal $s(\tau)$, and a dilated, shifted and normalized version of a mother wavelet $\psi(\tau)$, a complex valued function with particular properties (e.g. oscillating): $C W T(t, f)=\int s(\tau) f^{-1 / 2} \psi_{t, f}^{*}(\tau) d \tau$, where $*$ denotes complex conjugation. For our purpose, we use a Morlet wavelet. The 


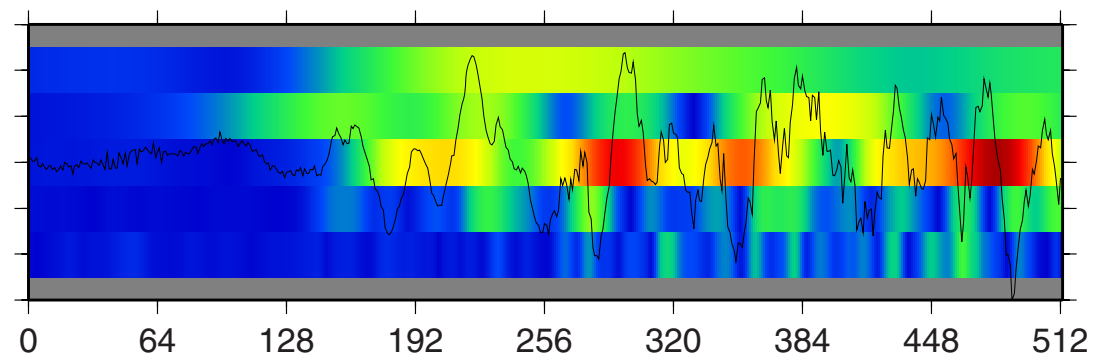

Fig. 1. Part of a seismogram (512 samples) showing a typical P-phase and its CWT coefficients. The coefficients have been normalized, and the level of gray corresponds to the size of the resulting coefficients. At the top the band with the lowest center frequency is shown $(0.25 \mathrm{~Hz})$ and at the bottom the highest frequency $(4 \mathrm{~Hz})$.

CWT is a measure of similarity between the basis functions and the signal itself. The CWT coefficients refer to the closeness of the signal to the basis function at the current scale $f$.

In practice, the continuous wavelet and integrals are approximated at discrete points in time and frequency. We employ the tool GWL [7] to produce the coefficients, i.e., for every (discrete) combination of $t$ and $f$, we obtain $C W T(t, f)$. The time scale for the CWT coefficients is discretized according to the original sampling rate $(20 \mathrm{~Hz})$ of the recorded time series $s(\tau)$. In the frequency domain, we consider $i=1 \ldots 5$ frequency bands on a dyadic scale, with center frequency $0.25 \mathrm{~Hz}, 0.5 \mathrm{~Hz}, 1 \mathrm{~Hz}, 2 \mathrm{~Hz}, 4 \mathrm{~Hz}$. The choice of those frequencies is due to the signal to noise ratio of seismic signals, which is fairly good in that range.

Figure 1 is an example of how the CWT of a seismic signal looks. We may observe the following: looking at single bands, there is no abrupt change in the level of gray, but rather there is a "bleeding"-effect - the gray transitions are smooth in the time dimension. Hence, there is an obvious dependence between the CWT coefficients in time. Across bands (scales) we observe a similar effect-large coefficients influence neighboring bands at the same time segment. This is the key to our main idea: 1) Consider the coefficients $C W T(t, f)$ as random variables 2) Exploit the Markov properties of those variables 3) Represent those Markov assumptions in a DBN.

\subsection{Relating the CWT Coefficients in a DBN}

Let $O_{i}^{t}=C W T(t, i)$ and introduce discrete state variables $U_{i}^{t}$ with binary states, $v=1,2$, corresponding to a large or small value of $O_{i}^{t}$. For each state of $U_{i}^{t}$, the withindistribution is defined to be a Gaussian. In graphical model terms, we may think of $U_{i}^{t}$ as a single binary parent node of $O_{i}^{t}$, that is $U_{i}^{t} \rightarrow O_{i}^{t}$, where $v=1,2: p\left(O_{i}^{t} \mid U_{i}^{t}=v\right)$ are Gaussian distributions. Hence, for each state the "members thereof" is subject to variation according to a Gaussian. Notice that the state variables $U_{i}^{t}$ are considered hidden. Marginalizing out this variable according to $U_{i}^{t} \rightarrow O_{i}^{t}$ yields a mixture with two Gaussian components, $\operatorname{Pr}\left(O_{i}^{t}\right)=\sum_{u_{i}^{t}} \operatorname{Pr}\left(O_{i}^{t}, u_{i}^{t}\right)=\sum_{v=1,2} p\left(O_{i}^{t} \mid U_{i}^{t}=v\right) p\left(U_{i}^{t}=\right.$ 
$v)$. For increasing $\left|\Omega_{U_{i}}\right|$ and adding the same number of conditional Gaussians to the mixture, we can, in fact, approximate any distribution arbitrarily close at the price of adding extra parameters to our model.

The observation about the dependence of the CWT coefficients is formulated in terms of $U_{i}^{t}$, i.e., the mixture weights. Hence, we may for any moment in time encode the dependence across bands in a BN with the graph $\forall t: U_{1}^{t} \rightarrow U_{2}^{t} \rightarrow U_{3}^{t} \rightarrow U_{4}^{t} \rightarrow U_{5}^{t}$ which means that $U_{i}^{t}$ is independent of all other variables given its neighbors in the graph, for all times. On the other hand, the time dependence between CWT coefficients along bands also gives rise to the graph $\forall i: U_{i}^{0} \rightarrow U_{i}^{1} \rightarrow U_{i}^{2} \rightarrow \ldots \rightarrow U_{i}^{T}$. We now create the graph of a DBN by combining the two graphs. The unrolled DBN graph of the (conditional) mixture weights becomes:

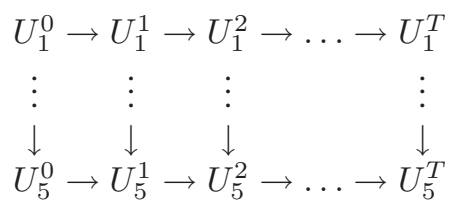

The CPDs $p\left(U_{i}^{t} \mid U_{i}^{t-1}, U_{i-1}^{t}\right)$ are the same for every time segment $t>0$, amounting to a total of 18 unique parameters. For time segment $t=0, p\left(U_{i}^{0} \mid U_{i-1}^{0}\right)$ are unique, amounting to 9 parameters. We take $\forall i: p\left(O_{i}^{0} \mid U_{i}^{0}\right)=\ldots=p\left(O_{i}^{T} \mid U_{i}^{T}\right)$ to be conditional Gaussians $\mathcal{N}\left(O_{i}^{t} \mid \mu_{i, v}, \sigma_{i, v}\right)$, for which we need to determine 20 parameters, $v=1,2, \forall i:\left\{\mu_{i, v}, \sigma_{i, v}\right\}$.

The dynamic range of the CWTs is very large, leading to numerous problems for parameter estimation and on-line classification. We propose to produce "more bounded" coefficients as follows. Extract the so-called average log-amplitude from the CWT coefficients, and associate $O_{i}^{t}$ with the residuals instead of the CWT coefficients $O_{i}^{t}=$ $\log C W T(t, i)-\bar{b}_{t}$ with $\bar{b}_{t}=\frac{1}{5} \sum_{i}^{5} \log C W T(t, i)$. By only retaining $O_{i}^{t}$ without the base-line $\bar{b}_{t}$ there is loss of information with respect to the original coefficients $C W T(t, i)$. We therefore exploit the expert-knowledge [1] captured in source spectra relating residuals to the average log-amplitude, i.e., a scaling relation between the frequency of the spectral maximum and the average log-amplitude. We model this domain knowledge characteristic explicitly in the DBN.

Add two extra binary variables to the structure, $U_{l o}^{t}=U_{6}^{t}$ and $U_{h i}^{t}=U_{7}^{t}$ and an extra continuous variable $O_{6}^{t}=O_{a m p}^{t}=\bar{b}_{t}$. We add deterministic arcs from the low frequency vertices $U_{1}^{t}, U_{2}^{t}$ and $U_{3}^{t}$ to $U_{l o}^{t}$ and from the high frequency vertices $U_{3}^{t}, U_{4}^{t}$ and $U_{5}^{t}$ to $U_{h i}^{t}$. These arcs define a mapping such that when any of $U_{1}^{t}, U_{2}^{t}$ or $U_{3}^{t}$ is in the state large then $U_{l o}^{t}$ is in the state large, otherwise it is in the state small; for high frequencies the same mapping to $U_{h i}^{t}$ holds. Finally add $\operatorname{arcs}$ from $U_{l o}^{t}$ and $U_{h i}^{t}$ to $O_{a m p}^{t}$. The associated CPDs are conditional Gaussians, one per parent set configuration $z=1, \ldots, 4: \mathcal{N}\left(O_{6}^{t} \mid \mu_{6, z}, \sigma_{6, z}\right)$, increasing the number of model parameters with 8 . Figure 2 shows the resulting DBN.

The DBN in figure 2 along with the (conditional) multinomial, Gaussian and deterministic CPDs was implemented in GMTK [2]. This tool allows for efficient inference and learning in DBNs. 


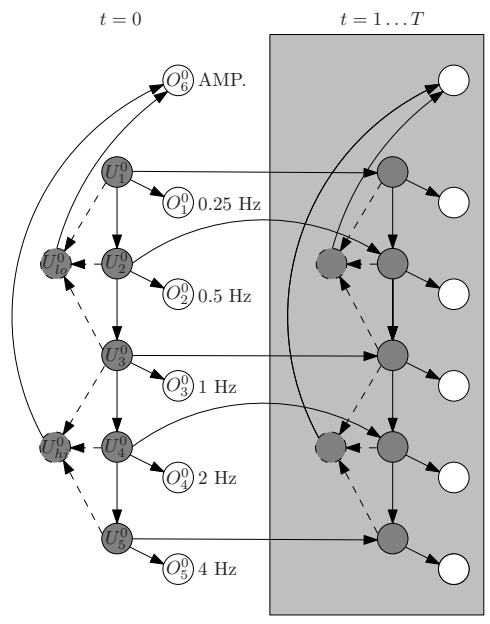

Fig. 2. The DBN capturing the relationships between the residuals, states, and the amplitude of a seismic pattern of length $T$. The dashed lines are deterministic relationships.

\section{Learning, Classification and Confidence}

The proposed DBN captures the intrinsic characteristics of a seismic pattern in a nondeterministic way by a joint distribution. We learn $C$ different patterns by quantifying $C$ different DBNs, that is, DBNs with the same model structure but different parameters, $\boldsymbol{\theta}_{1}, \ldots, \boldsymbol{\theta}_{C}$. A previously unseen pattern $\boldsymbol{y}=\left(\boldsymbol{y}^{0}, \ldots, \boldsymbol{y}^{T}\right)$ is classified as $I=\arg \max _{i} \operatorname{Pr}\left(\boldsymbol{\theta}_{i} \mid \boldsymbol{y}\right)=\arg \max _{i} \operatorname{Pr}\left(\boldsymbol{y} \mid \boldsymbol{\theta}_{i}\right)=\arg \max _{i} \log \operatorname{Pr}\left(\boldsymbol{y} \mid \boldsymbol{\theta}_{i}\right)$ where the 1st equality follows from $\operatorname{Pr}\left(\boldsymbol{\theta}_{i} \mid \boldsymbol{y}\right) \propto \operatorname{Pr}\left(\boldsymbol{y} \mid \boldsymbol{\theta}_{i}\right)$ under the assumption of a uniform prior over the different DBNs. Since $\boldsymbol{y}$ consists of observed variables, computing $\operatorname{Pr}\left(\boldsymbol{y} \mid \boldsymbol{\theta}_{i}\right)$ entails marginalizing the hidden state variables out of the joint distribution represented as our DBN, $\operatorname{Pr}\left(\boldsymbol{y}^{0}, \ldots, \boldsymbol{y}^{T} \mid \boldsymbol{\theta}_{i}\right)=\sum \boldsymbol{u} \operatorname{Pr}\left(\boldsymbol{y}^{0}, \boldsymbol{u}^{0} \ldots, \boldsymbol{y}^{T}, \boldsymbol{u}^{T} \mid \boldsymbol{\theta}_{i}\right)$. Rather than summing out the hidden variables directly, which would be intractable in general, the algorithm for efficient DBN inference as described in [13] is called via GMTK.

Assigning $\boldsymbol{y}$ to the "winning" class unfortunately gives no indication about the confidence of the assignment: Is the decision close to the boundary of the other classes? For a binary classification problem the following ratio provides a measure of confidence of assigning $\boldsymbol{y}$ to $I$ rather than to the alternative $\bar{I}, \operatorname{Pr}\left(\boldsymbol{y} \mid \boldsymbol{\theta}_{I}\right) / \operatorname{Pr}\left(\boldsymbol{y} \mid \boldsymbol{\theta}_{\bar{I}}\right)$. For multiclass problems, we define this to be the geometric mean of every alternative (inferior) model $\sqrt[C-1]{\prod_{i \neq I}^{C} \frac{\operatorname{Pr}\left(\boldsymbol{y} \mid \boldsymbol{\theta}_{I}\right)}{\operatorname{Pr}\left(\boldsymbol{y} \mid \boldsymbol{\theta}_{i}\right)}}=\operatorname{Pr}\left(\boldsymbol{y} \mid \boldsymbol{\theta}_{I}\right) \cdot \prod_{i \neq I}^{C} \operatorname{Pr}\left(\boldsymbol{y} \mid \boldsymbol{\theta}_{i}\right)^{-\frac{1}{C-1}}$. We take the logarithm of this expression to avoid numerical problems. A value of 0 then means that the classifier is indifferent; a large value means that with high confidence $I$ is correct.

Training of the DBNs amounts to computing the Maximum Likelihood Estimates (MLE) of $\boldsymbol{\theta}_{1}, \ldots, \boldsymbol{\theta}_{C} \cdot C$ training sets are provided, each set with examples of patterns belonging to the class in question. MLEs are computed via (G)EM [9] using GMTK.

Classification of continuous time-series $s(\tau)$ starting at time $\tau$ is done in a slidingwindow fashion. A pattern captured by a DBN may last for $T$ time slices ( $T$ may vary 
Table 1. Results using 4-fold cross-validation. The columns "Acc. -Amp" and "Acc. +Amp" are the accuracy results with and without the amplitude feature. The remaining columns are statistics for the DBNs with the amplitude feature. The number between brackets is the number of "signal" examples for the station. The number of "noise" examples is the same.

\begin{tabular}{l|r||r||r|r|r||r|r|r} 
Station & Acc. - Amp. & Acc. + Amp. & FP rate & Conf. FP & Conf. TP & FN rate & Conf. FN & Conf. TN \\
\hline anto (27) & 0.87 & 0.91 & 0.071 & 102 & 2004 & 0.107 & 92 & 453 \\
aqu (30) & 0.80 & 0.92 & 0.031 & 106 & 1744 & 0.125 & 163 & 411 \\
arg (35) & 0.86 & 0.94 & 0.065 & 227 & 3754 & 0.025 & 75 & 658 \\
cey (32) & 0.86 & 0.96 & 0.055 & 103 & 3314 & 0.056 & 232 & 585 \\
css (27) & 0.81 & 0.98 & 0.050 & 96 & 2333 & 0.028 & 104 & 554 \\
itm (35) & 0.91 & 0.93 & 0.051 & 346 & 7546 & 0 & - & 506 \\
kek (32) & 0.86 & 0.91 & 0.098 & 590 & 4504 & 0.032 & 124 & 435 \\
psz (33) & 0.92 & 0.98 & 0.031 & 40 & 1238 & 0 & - & 635 \\
rdo (35) & 0.98 & 1 & 0 & - & 5168 & 0 & - & 590 \\
\hline & 0.87 & 0.95 & 0.050 & 201 & 3511 & 0.041 & 131 & 536
\end{tabular}

per pattern), i.e., the CWT coefficients are computed from $s(\tau+1), \ldots, s(\tau+T)$, the residuals and the average log-amplitude are extracted, DBN inference is performed, and the window is moved to $\tau:=T$, and so on. The computational burden is insignificant: computing the CWT can be done very quickly, and the actual classification, which entails inference in $C$ (sparse) DBNs, can be done very fast as well.

\section{Experiments}

We performed experiments using seismic data recorded by the European broadband network. We considered 9 individual stations. For now, we are primarily interested in distinguishing seismic signal from noise (ambient, urban, etc). We learn the typical pattern of signal from the transient signal onset of a seismogram, i.e., the so-called $P$ phase, lasting for 512 time slices. For each station these phases were picked by hand by a seismologist (domain expert). Similarly, several examples of noise were selected before the P-phase (also lasting for 512 slices).

The performance was tested using 4-fold cross-validation for each station using single patterns. In order to see if the amplitude feature makes a difference when classifying, we ran tests with and without this attribute. Table 1 shows the results. As we may see, including the amplitude indeed improves classification accuracy. In general, the accuracy is high, especially when taking into consideration the relatively small training instances provided. We are primarily concerned with the false positives (presented as noise, but classified as signal) for which the false positive rate has been computed too. How far are these false alarms from being classified correctly as negatives? To get an impression of that, we compute the average confidence of the false positives. We see that they are close to the decision boundary, especially when we compare to the average confidence of the true positives. This means that when the classifier is issuing a false alarm, it has been triggered by a pattern which is far from being a "typical" non-signal. We do the same for the false negatives (presented as positives, classified as negatives). The 

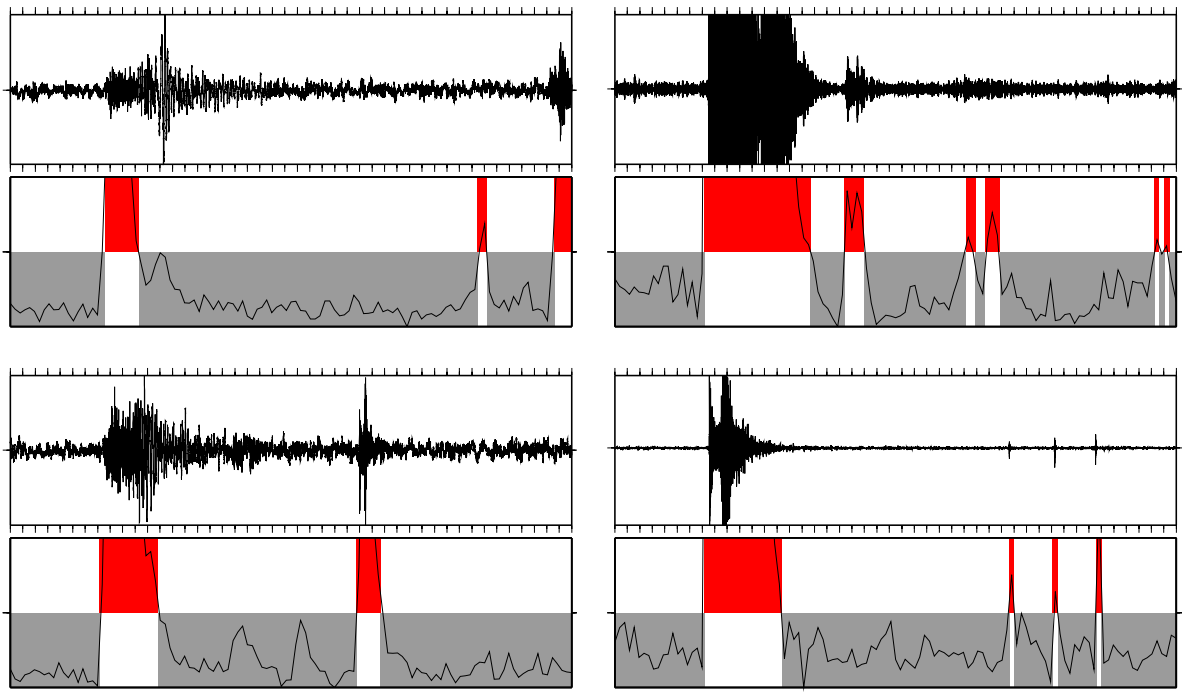

Fig. 3. Four seismograms lasting for 30 minutes. At the top the unfiltered traces and below the real-time classifications are shown together with the plot referring to the confidence of the class assignment with respect to the alternative.

average confidence of a false negative is pretty close to the decision boundary compared to the average true negatives.

The detection of weak seismic signals is difficult, even for the trained seismologist. Presenting the measure of confidence during real-time classification allows the seismologist to intervene: weak (uncertain) false alarms of missed events can be corrected.

Figure 3 shows four examples of seismograms and below each trace the classification and confidence plots are given. The horizontal line in the middle of the confidence plots is the decision boundary between classes. If the plot is above the threshold, the pattern is classified as signal, if it is below it is noise. Every 15 th second a classification and confidence is given of the previous 15 seconds. To the untrained eye there seems to be several misclassifications, but in fact all patterns detected were correct signal classifications according to the domain expert. Although the examples might indicate that the amplitude of the seismic signals provides enough information for classification, this is not the case; certainly the amplitude plays a role, but other factors are important too as suggested by the accuracy results in table 1 (with and without the amplitude).

\section{Conclusion}

We have presented a novel method for real-time classification of seismic patterns using DBNs. The symbolic DBN modeling approach gives rise to a model with a limited number of parameters which is crucial because only a small number of training samples exist per station. Experimentally the accuracy and the error rates are good, taking into consideration that the decision problem is very fuzzy in nature. Moreover, the 
confidence measure provides the seismologist with information about the closeness of the signal to the assigned class.

Since our method is very generic, future works goes in the direction to extend the classification to more detailed modeling of the "anatomy of seismograms", i.e., the distinction of individual seismic phases within an earthquake signal that allow seismologists to study earthquake source processes and effects of seismic wave propagation. The actual implementation of the system at selected stations of the European broadband network will be realized in near future.

\section{Acknowledgments}

We would like to acknowledge Andreas Köhler for preparing the data and Michail Kulesh for letting us use GWL. This work has been made possible under the EC-Project NERIES contract no. 026130.

\section{References}

1. Aki, K., Richards, P.G.: Quantitative Seismology. University Science Books (2002)

2. Bilmes, J., Zweig, G.: The graphical models toolkit: An open source software system for speech and time-series processing. In: Intl. Conf. on Acoustics, Speech and Signal Proc (2002)

3. Chui, C.K.: An Introduction to Wavelets. Academic Press, London (1992)

4. Dean, T., Kanazawa, K.: A model for reasoning about persistence and causation. Artificial Intelligence 93(1-2), 1-27 (1989)

5. Gendron, P., Ebel, J., Manolakis, D.: Rapid Joint Detection and Classification with Wavelet Bases via Bayes Theorem. Bull. Seism. Soc. Am. 90(3), 764-774 (2000)

6. Joswig, M.: Pattern recognition for earthquake detection. Bull. Seism. Soc. Am. 80(1), 170186 (1990)

7. Kulesh, M., Holschneider, M., Diallo, M.S.: Geophysics wavelet library: Applications of the continuous wavelet transform to the polarization and dispersion analysis of signals. Computers \& Geoscience (Submitted 2007)

8. Kumar, P., Foufoula-Georgiou, E.: Wavelet analysis for geophysical applications. Reviews of Geophysics 35(4), 385-409 (1997)

9. Lauritzen, S.L.: The EM algorithm for graphical association models with missing data. Computational Statistics and Data Analysis 19, 191-201 (1995)

10. Okada, Y., Kasahara, K., Hori, S., Obara, K., Sekiguchi, S., Fujiwara, H., Yamamoto, A.: Recent progress of seismic observation networks in Japan-Hi-net, F-net, K-NET and KiKnet. Earth, Planets, and Space, 56:D15+ (August 2004)

11. Pearl, J.: Probabilistic Reasoning in Intelligent Systems: Networks of Plausible Inference. Morgan Kaufmann, San Francisco (1988)

12. Withers, M., Aster, R., Young, C., Beiriger, J., Harris, M., Moore, S., Trujillo, J.: A comparison of select trigger algorithms for automated global seismic phase and event detection. Bull. Seism. Soc. Am. 88(1), 95-106 (1998)

13. Zweig, G., Russell, S.: Speech Recognition with Dynamic Bayesian Networks. In: AAAI, pp. 173-180 (1998) 